\title{
Morbidität und Medikation begünstigen Pneumonie bei alten Menschen
}

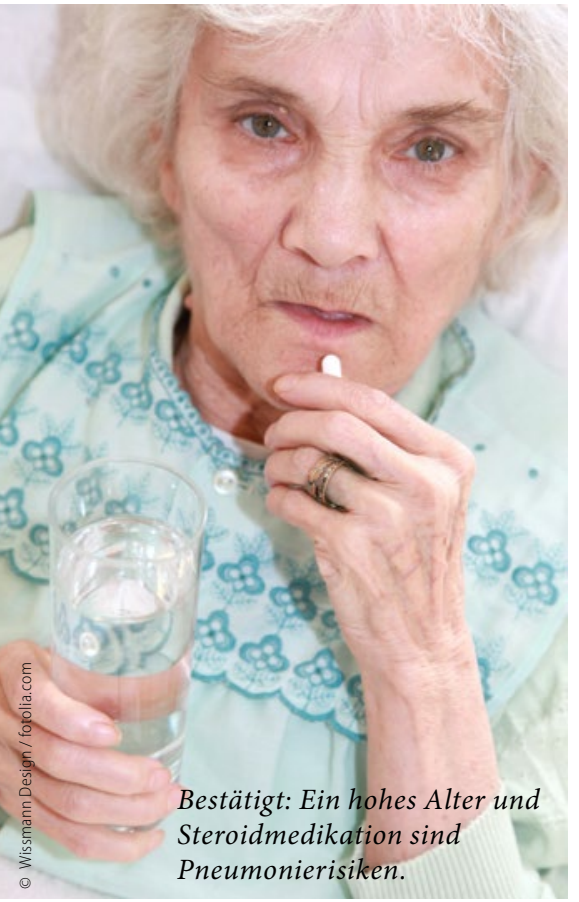

Hintergrund und Fragestellung: Da die ambulant erworbene Pneumonie (CAP) die häufigste Infektionserkrankung ist und im hohen Lebensalter mit einer hohen Morbidität und Mortalität vergesellschaftet ist, wollen die Autoren in ihrer Arbeit Prognoseindices entwickeln, um das Zweijahres-Risiko einer Pneumonie bei alten Menschen bestimmen zu können.

Patienten und Methoden: Es handelt sich um eine retrospektive Kohortenstudie bei über 65-jährigen, im eigenen Wohnumfeld lebenden, nicht dementen Menschen, die Mitglieder der integrierten Gesundheitsvorsorge Group Health $(\mathrm{GH})$ sind. Potenzielle Risikofaktoren

für eine Pneumonie wurden über a) Fragebögen und Interviews b) funktionelle Untersuchungen (Handkraft, Gehgeschwindigkeit, usw.) und c) administrative Routinedaten der Gesundheitsfürsorge erhoben. Die Inzidenz der ambulant erworbenen Pneumonie wurde aus den Patientenkrankenakten ermittelt.

Ergebnisse: Bei 3.375 Studienteilnehmern wurden in 12.998 Untersuchungen 642 ambulant erworbene Pneumonien detektiert. Alter, Geschlecht, COPD, Herzinsuffizienz, inhalative/orale Kortikosteroideinnahme wurden als Risikofaktoren identifiziert. Diese Risikofaktoren sind auch in den Patientenakten verfügbar (electronic medical records-EMR) und haben dieselbe Vorhersagegüte wie aufwändige Daten, beispielsweise die Erhebung des funktionalen Status (c-index 0,69).

Originalie

Jackson ML, Walker R, Lee $S$ et al. Predicting 2-Year Risk of Developing Pneumonia in Older Adults without Dementia. JAGS 2016:64:1439-47.
Schlussfolgerung: Mithilfe medizinischer Informationen die über die Routinedaten der Gesundheitsversicherer abgefragt werden können, lässt sich das Zweijahres-Risiko für eine Pneumonie abschätzen.

\section{- Kommentar von Univ.- Prof. Dr. med. Hans Jürgen Heppner}

\section{Basis für präzisere Empfehlungen zur Pneumokokkenimpfung?}

Die Inzidenz der ambulant erworbenen Pneumonie mit der Folge einer stationären Klinikaufnahme liegt bei den ältesten Erwachsenen, mit 164,3 Fällen pro 10.000 Personen in einem Alter von 80 Jahren und älter, am höchsten [1]. Die Autoren haben daher klinisch relevante Überlegungen angestellt, um eine Risikopopulation zu identifizieren. $53 \%$ der untersuchten Kohorte waren 70 bis 80 Jahre und damit genau die geriatrische Zielpopulation. Die benannten Risikofaktoren sind bekannt $[2,3]$. Jedoch überrascht es, dass die Routinedaten eines Krankenversicherers eine ebenso zuverlässige Vorherhersage erlauben wie die Erhebung des funktionellen Status der Studienpopulation. Hier bleibt zu hinterfragen, ob dies so einfach auf deutsche Verhältnisse zu übertragen ist. Auch ist die Zielsetzung zu betrachten, ob man einen "schnellen“ klinischen Risikoscore erheben will oder detaillierte Angaben für wissenschaftliche Fragestellungen benötigt. Somit resultieren folgende Überlegungen: a) Wenn sich die Zuverlässigkeit bestätigt, könnte der Risikoscore aus den Routinedaten automatisch erhoben und Präventionsmaßnahmen eingeleitet werden und b) Da Pneumokokken die Haupterreger der ambulant erworbenen Pneumonie sind, lässt sich aus den Risikoergebnissen eine Empfehlung zur Pneumokokkenimpfung ableiten.
Literatur

1. Jain S, Self WH, Wunderink RG et al. Community-Acquired Pneumonia Requiring Hospitalization among U.S. Adults. N Engl J Med 2015;373:415-27.

2. Jackson ML, Nelson JC, Jackson LA. Risk factors for community-acquired pneumonia in immunocompetent seniors. J Am Geriatr Soc 2009;57:882-8.

3. LaCroix AZ, Lipson S, Miles TP et al. Prospective study of pneumonia hospitalizations and mortality of U.S. older people: The role of chronic conditions, health behaviors, and nutritional status. Public Health Rep 1989;104:350-60.

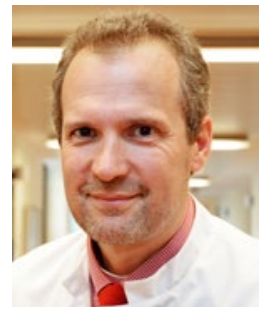

Univ.- Prof. Dr. med. Hans Jürgen Heppner, MHBA

Chefarzt Geriatrische Klinik und Tagesklinik Lehrstuhl für Geriatrie Universität Witten/Herdecke Dr.-Moeller-Straße 15, 58332 Schwelm hans.heppner@uni-wh.de 